\title{
Solid pseudopapillary tumor of pancreas
}

\author{
Ali Aminian · Rasoul Mirsharifi · Sima Moulavi · Habibollah Dashti · Fouzeyah A. H. Ali • Abbas Alibakhshi
}

Received: 18 April 2008 / Accepted: 27 April 2009

(C) Association of Surgeons of India 2010

A 17-year-old woman presented with progressive abdominal swelling since 8 months back after cesarean section. There was no history of previous medical and surgical problems including trauma. The only positive finding in physical examination was the presence of a large fixed epigastric abdominal mass. Routine laboratory tests, amylase, tumor markers and hydatid serology were in normal limits. CT scan showed a large cystic abdominal lesion with irregular margins (Fig. 1). Exploratory laparotomy revealed a cystic lesion in the head of pancreas. Pancreaticoduodenectomy was performed successfully. Histopathologic examination was in favor of solid pseudopapillary tumor of pancreas.

In approach to cystic pancreatic lesions, the major challenge is distinguishing pseudocysts and serous cystadenomas from the other, malignant or potentially malignant, types of cystic tumors. A recent history of pancreatitis, enzyme-rich fluid in the cyst, and communication between the cyst and the pancreatic duct suggest that the cystic lesion is a postpancreatitic pseudocyst. Serous cystadenomas, diagnosed by finding glycogen-rich cells on fine-needle aspiration biopsy, can be followed without resection if they are not symptomatic $[1,2]$.

However, the presence of a solid component, septations or nodular irregularities of the cyst wall, mucin in the cyst, mucin-secreting cells on biopsy, a high cyst fluid viscosity, or a high cyst fluid carcinoembryonic antigen is suggestive of a potentially malignant tumor $[1,2]$.

\footnotetext{
A. Aminian ${ }^{1} \cdot$ R. Mirsharifi ${ }^{1}$ S. Moulavi ${ }^{1} \cdot$ H. Dashti ${ }^{1}$.

F. A. H. Ali ${ }^{2}$ A. Alibakhshi ${ }^{1}$

${ }^{1}$ Department of General Surgery,

Tehran University of Medical Sciences, Tehran, Iran

${ }^{2}$ Department of Radiology,

Mubarak Al-Kabeer Hospital, Kuwait
}

A. Aminian $(\bowtie)$
E-mail: aliaminian@doctor.com

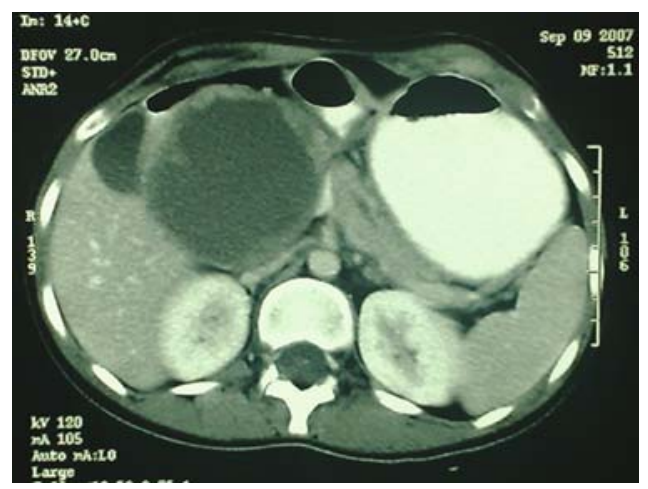

Fig. 1 CT scan: A large cystic abdominal lesion with irregular margins

All pancreatic cysts should be resected unless the diagnosis of pseudocyst or serous cystadenoma can be made with certainty. For those located in the pancreatic tail, a distal pancreatectomy is ideal, whereas cystic lesions in the pancreatic head would require pancreaticoduodenectomy $[1,2]$.

Solid pseudopapillary tumor, a relatively uncommon and potentially malignant tumor, often occurs in young women. The tumors are usually large, round, and well-demarcated masses that can occur in any part of the pancreas. Local resection is usually curative, although incomplete removal can result in local recurrence $[1,2]$.

\section{References}

1. Papavramidis T, Papavramidis S (2005) Solid pseudopapillary tumors of the pancreas: Review of 718 patients reported in English literature. J Am Coll Surg 6:965-972

2. D'Souza M, Abraham P, Shah SR (2006) Solid-cystic papillary tumor of the pancreas: A missed diagnosis. Indian J Surg 68:269-271 